\title{
Superconducting single-charge transistor in a tunable dissipative environment
}

\author{
Frank K. Wilhelm ${ }^{1,2,3}$, Gerd Schön ${ }^{1,4}$, Gergely T. Zimányi ${ }^{2}$ \\ ${ }^{1}$ Institut für Theoretische Festkörperphysik, Universität Karlsruhe, D-76128 Karlsruhe, Germany. \\ ${ }^{2}$ Department of Physics, UC Davis, Davis CA 95616, U.S.A \\ ${ }^{3}$ Quantum Transport Group, TU Delft, P.O. Box 5046, 2600 GA Delft, The Netherlands \\ ${ }^{4}$ Forschungszentrum Karlsruhe, Institut für Nanotechnologie, D-76021 Karlsruhe, Germany
}

\begin{abstract}
We study a superconducting single-charge transistor, where the coherence of Cooper pair tunneling is destroyed by the coupling to a tunable dissipative environment. Sequential tunneling and cotunneling processes are analyzed to construct the shape of the conductance peaks and their dependence on the dissipation and temperature. Unexpected features are found due to a cross-over between two distinct regimes, one 'environment-assisted' the other 'environment-dominated'. Several of the predictions have been confirmed by recent experiments. The model and results apply also to the dynamics of Josephson junction quantum bits on a conducting ground plane, thus explaining the influence of dissipation on the coherence.
\end{abstract}

The behavior of a quantum system coupled to a dissipative environment is one of the paradigms of modern physics [1]. It is the central challenge in any attempt to build and manipulate quantum information systems, since fluctuations and dissipation limit the quantum coherence of the device. For instance, for qubits based on two different charge states of a Josephson junction [2] one important source of dissipation are the normal electrons in the ground plane. A conducting ground plane is an appropriate tool however, to compensate for the random offset charges of the superconducting island. In the experimental realization of Nakamura et al. [3] this was achieved by mounting the device on a gold ground plane.

In earlier experiments the Berkeley group [4 had mounted an array of Josephson junctions on a twodimensional electron gas (2DEG), separated from it by an insulating layer. In this setup the density of normal electrons in the 2DEG, and hence the source and strength of the dissipation, can be tuned by a back gate over a wide range (see also [5]). This influences the collective properties of the array in an qualitative way [6]. In more recent experiments [7] the Berkeley group investigated the transport properties of a Superconducting Single-Charge Transistor (SSCT) coupled to a tunable 2DEG (see Fig. 1). They observed an unexpected dependence of the conductance peak heights and widths on temperature and dissipation.

In previous work the nonlinear current branch of a SSCT has been studied 810. Due to the coupling of the SSCT to the metallic ground plane the Cooper pair charges interact with normal metal image charges, which turns the tunneling of Cooper pairs dissipative. A central result of the present paper is the identification and characterization of the previously uninvestigated normal current branch in the linear and nonlinear response regime. We determine how the conductance depends on the gate and transport voltage, environment conductance and temperature. We also provide estimates how dissipation limits the quantum oscillations of qubits.
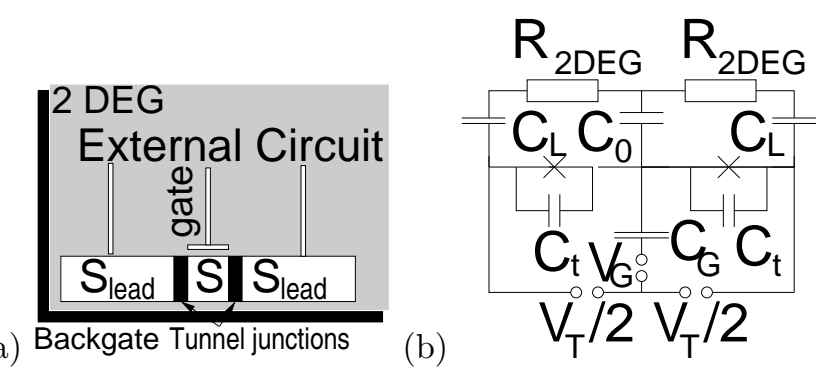

FIG. 1. (a) Top view of the system under consideration. The 2DEG is in the substrate. (b) Equivalent circuit.

In the following we concentrate on systems (see Fig. 1) with low resistance per square of the ground plane, $R \ll$ $h / 4 e^{2} \approx 6.5 \mathrm{k} \Omega$, and we choose the gate and junction capacitances, $C_{\mathrm{g}}$ and $C_{\mathrm{j}}$, and the capacitances between the island and the leads to the metallic plane, $C_{0}$ and $C_{1}$, to be ordered as follows: $C_{\mathrm{g}}, C_{\mathrm{j}} \ll C_{0} \ll C_{\mathrm{l}}$. The effects of the dissipative metallic ground plane on the SSCT are captured by the real part of the impedance,

$$
\operatorname{Re}\{Z(\omega)\}=R /\left[1+\left(\omega R C_{\mathrm{j}}\right)^{2}\right] .
$$

Interestingly, even though the different capacitances introduce several frequency regimes, in all regimes $\operatorname{Re} Z(\omega)$ is determined by the capacitance of the tunnel junction $C_{\mathrm{j}}$ only. Quasiparticle dissipation may be ignored at temperatures much below the superconducting gap.

We construct our theory following Ref. 11, 12]. The environment can be described by a harmonic oscillator bath. An integration over the quadratic bath degrees of freedom is possible. The resulting sequential tunneling rate through one junction [13],

$$
\Gamma\left(\delta E_{\mathrm{ch}}\right)=(\pi / 2 \hbar) E_{\mathrm{J}}^{2} P\left(\delta E_{\mathrm{ch}}\right),
$$

depends on the change in charging energy during the tunneling process, which in turn depends on the gate voltage $n_{\mathrm{g}}=V_{\mathrm{g}} C_{\mathrm{g}} / e$ and the transport voltage $V_{\mathrm{tr}}$. For a process 

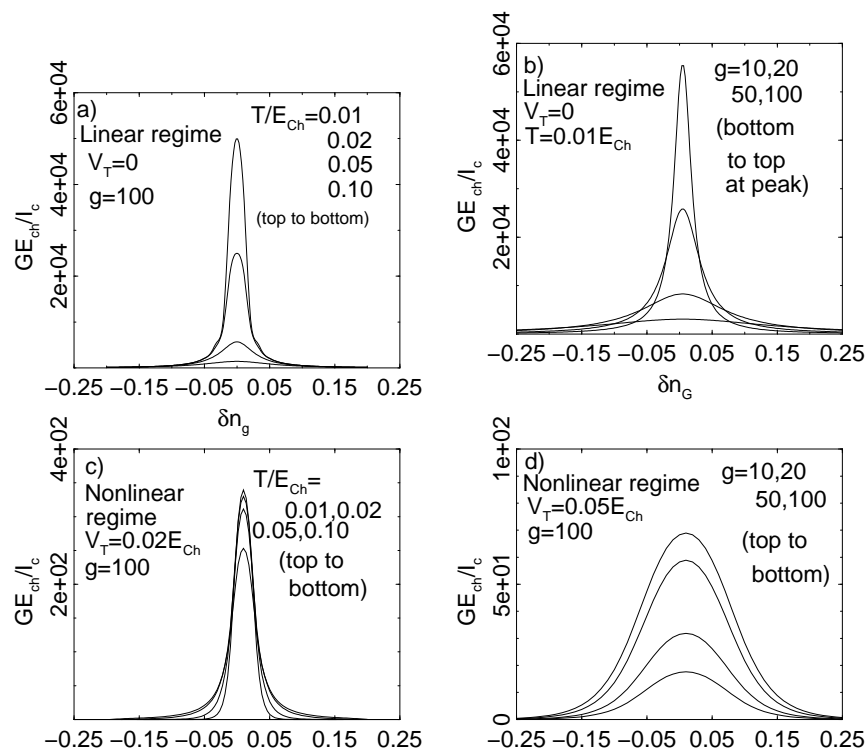

FIG. 2. Condectance peaks in lowest order (\$nequential tunneling) scaled by the critical current of a single junction, $I_{c}$ and the charging energy $E_{\mathrm{C}}$ of the island, assuming $C_{\mathrm{G}} \ll C_{\mathrm{j}}$.

which increases the number of excess Cooper pairs from $n$ to $n+1$ is given by

$$
\delta E_{\mathrm{ch}}=-4 E_{\mathrm{C}}\left(2 n-n_{\mathrm{G}}+1\right)+e V_{\mathrm{tr}} .
$$

The (total) capacitance of the island determines the energy scale $E_{\mathrm{C}}=e^{2} / 2\left(2 C_{\mathrm{j}}+C_{\mathrm{g}}\right)$, while $E_{\mathrm{J}}$ is the Josephson coupling energy of the tunnel junctions (here assumed to be equal). The function $P(E)$ can be expressed by a correlation function $K(t)=$ $\frac{4 e^{2}}{h} \int_{-\infty}^{\infty} \frac{d \omega}{\omega} \operatorname{Re}\{Z(\omega)\}\left\{\operatorname{coth}\left(\frac{\hbar \omega}{2 T}\right)[\cos (\omega t)-1]-i \sin (\omega t)\right\}$ via $P(E)=\frac{1}{2 \pi \hbar} \int_{-\infty}^{\infty} d t \exp \left[K(t)+i \frac{E t}{\hbar}\right]$.

In the situation considered, $K(t)$ can be evaluated analytically and in the long-time limit, $|t| \gg R C_{\mathrm{j}}$, it reads

$$
\begin{aligned}
K(t)= & -\frac{2}{g}\left[\pi T|t|+\log \left(1-e^{-2 \pi T|t|}\right)\right. \\
& \left.+\gamma+\log \left(2 \pi T R C_{\mathrm{j}}\right)+i \operatorname{sign}(t) \frac{\pi}{2}\right] .
\end{aligned}
$$

In the low energy regime, $E, T \ll R C_{\mathrm{j}}$, we find

$$
P(E)=\frac{\left(2 \pi e^{-\gamma} T R C_{\mathrm{j}}\right)^{\frac{2}{g}}}{2 \pi^{2} T} \operatorname{Re}\left[e^{-i \frac{\pi}{g}} B\left(\frac{1}{g}-\frac{i E}{2 \pi T}, 1-\frac{2}{g}\right)\right]
$$

where $\gamma=0.577 \ldots, g=h / 4 e^{2} R$ is the dimensionless conductance of the ground plane, and $B(x, y)$ the Beta function. We observe that the energy appears only in the dimensionless combination $\epsilon=E g / 2 \pi T$.

Given the tunneling rates $\Gamma\left(\delta E_{\mathrm{ch}}\right)$ we construct a master equation and determine the current $I$ through the SSCT. The difference of the charging energies $\delta E_{\mathrm{ch}}$ varies as the gate voltage is tuned. At low temperatures, for most values of $n_{\mathrm{g}}$, the large cost in charging energy suppresses transport by the Coulomb blockade. At the degeneracy points of the states with $n$ and $n+1$ Cooper pairs (i.e. $\delta E_{\mathrm{ch}}=0$ ), transport becomes possible. Thus the conductance exhibits Coulomb oscillations as a function of the gate voltage.

Previously published work 12,14] concentrated on the low temperature regime $\epsilon \gg 1$. Here the asymptotic expansion of Eq. (5) yields $P(E) \approx 2 / g E$ [11]. For instance at the degeneracy point, we have $\delta E_{\mathrm{ch}}=e V_{\mathrm{tr}}$ and the $I$ $V$ characteristics is strongly nonlinear

$$
I=4 \pi E_{\mathrm{J}}^{2} / \hbar g V_{\mathrm{tr}} .
$$

Away from the degeneracy point the high energy cost, $\delta E_{\text {ch }} \gg T$, suppresses tunneling (Coulomb blockade). Thus this regime is still described by $|\epsilon| \gg 1$. Detailed balance requires $P(-E)=e^{-E / T} P(E)$, from which one finds that the conductance is suppressed by an exponential activation factor [1].

A closer inspection shows, however, that for typical transport voltages of $V_{\mathrm{tr}} \approx 1 \mathrm{nV}$, even at the lowest temperatures of $T=20 \mathrm{mK}$, for the typical values of $g=1 . .100$ one has $\epsilon \ll 1$. This motivated us to study this previously ignored parameter regime. Surprisingly we find that both at the peaks and in the Coulomb blockade regime the system's response becomes linear, although for different reasons.

First, at the peaks and for low transport voltages $\delta E_{\mathrm{ch}}=e V_{\mathrm{tr}} \ll T$ one can expand Eq. (可). We find that in this regime the current response is linear

$$
I\left(V_{\mathrm{tr}}, n_{\mathrm{G}}=n+1\right)=e \pi E_{\mathrm{J}}^{2} g e V_{\mathrm{tr}} / \hbar T^{2},
$$

and the height of the conductance peak now increases with increasing conductance $g$ of the metallic plane, in contrast to what could be concluded from Eq. (6).

The full shape of the conductance peak derived from Eq. (5) is shown in Figure 2. At low enough transport voltages, $V_{\mathrm{tr}}<2 \pi T / g$, at the peaks we have $\epsilon<1$ and the current response is linear and transport is provided by 'environment-dominated' sequential tunneling (ED$\mathrm{ST}$ ). The peak height is proportional to $1 / T^{2}$ at fixed $g$ (Fig. 2(a)) and proportional to $g$ at fixed $T$ (Fig. 2(b)). Away from the peak the energy change $\delta E_{\mathrm{ch}}$ for a tunneling process is large even for $V_{\mathrm{tr}}=0$. Therefore one crosses over to the regime $\epsilon \gg 1$. Here the differential conductance at some fixed voltage is proportional to $1 / g$. This tendency is opposite to that of the peaks, and manifests itself in the crossing of the conductance $G\left(V_{\mathrm{g}}\right)$ curves in the tails (see Fig. 2(b)). In this 'environmentassisted' sequential tunneling (EA-ST) regime, the conductance may still be appreciable; it is reduced due to Coulomb blockade only for $\delta E_{\mathrm{ch}}>T$, i.e. at $\epsilon>g$. We also find that the width of the peak increases with decreasing $g$, and the conductance in the tails decreases with increasing $T$. 
In a transport experiment the voltage is necessarily finite, $V_{\text {tr }}>0$. At low enough temperatures $T \ll V_{\text {tr }} g / 2 \pi$ this forces the system to $\epsilon>1$, where the nonlinear response formulae govern the physics. Thus, the apparent divergence of the conductance at the peak, $G \simeq 1 / T^{2}$ is regularized by the transition to the nonlinear regime, yielding a finite conductance for $T \rightarrow 0$ (Fig. 2(c)). Also, at sufficiently large $V_{\mathrm{tr}}$ the conductance monotonically increases with decreasing $g$ over the whole energy range (Fig. 2(d)).

In second order in $E_{\mathrm{J}} / E_{\mathrm{ch}}$ the current is exponentially suppressed in the Coulomb blockade regime. Here, higher order terms, such as the fourth order cotunneling process, may yield important contributions. Cotunneling describes the simultaneous coherent transfer of two Cooper pairs, where the the energetically forbidden intermediate state is occupied only virtually. As a consequence the process is not suppressed by an exponential activation factor in the blockade regime. The most relevant term (for $\delta E_{\mathrm{ch}} \ll-T / g$ ) leads to the rate [15]

$$
\Gamma_{\mathrm{CT}}=\frac{E_{J}^{4}}{2 \pi \delta E_{\mathrm{ch}}^{2} T} \operatorname{Re}\left[e^{-4 i \pi / g} B\left(-i \frac{V_{\mathrm{tr}}}{2 \pi T}+\frac{4}{g}, 1-\frac{8}{g}\right)\right] .
$$

At low voltages we thus find again a linear response regime with a decay rate $\Gamma_{\mathrm{CT}} \propto V_{\mathrm{tr}} g / T^{2}$.

Combining these results, we can read off the peak width within linear response. Depending on the experimental conditions, the conductance is dominated by environment-assisted sequential tunneling (EA-ST) or environment-dominated cotunneling (ED-CT). This leads to a half-width of $\delta E_{\mathrm{ch}, 1 / 2} \propto \max \left(T / g, E_{\mathrm{J}}\right)$ whereas a fixed off-peak conductance $g_{0}$ is reached at $\delta E_{\mathrm{ch}, \mathrm{g}_{0}} \propto \max \left(E_{\mathrm{J}} / \sqrt{g g_{0}}, \sqrt{g / g_{0}} E_{\mathrm{J}}^{2} / T\right)$. At high transport voltages and large $\delta E_{\mathrm{ch}}$ the response becomes nonlinear, yielding $\Gamma_{\mathrm{CT}} \propto 1 / g V^{2}$. Remarkably however the ED-ST regime extents to large $V_{\mathrm{tr}}$, in the region where the bare charging energy difference and $V_{\mathrm{tr}}$ combine to a small net $\delta E_{\mathrm{ch}}$, generating again a low value of $|\epsilon| \ll 1$. Here again the incoherent transport of Cooper pairs dominates, with a conductance $G \propto g E_{J}^{2} / T^{2}$. The expressions for the conductances in the different parameter regimes are summarized in Fig. 3.

So far we considered relatively short and wide leads, when the bilayer formed by the leads and the metallic plane can be described as a capacitor. If the leads are longer and narrower, the spatial correlations of charges moving within the bilayer modify the fluctuation spectruma and should be taken into account. Then the proper model for the leads is an RC transmission line using the per-square capacitance and resistance, rather than the $C_{\mathrm{L}}$-capacitors in Fig. 1 . The impedance of such lines is $Z_{R C}(\omega)=\sqrt{R / i \omega C_{\mathrm{sq}}}$ where $C_{\mathrm{sq}}$ is the square capacitance between the lead and the ground plane. In this case the $\omega^{-1 / 2}$ divergence governs the impedance. At $T=0$ the model has been analysed before [14,12]. Here

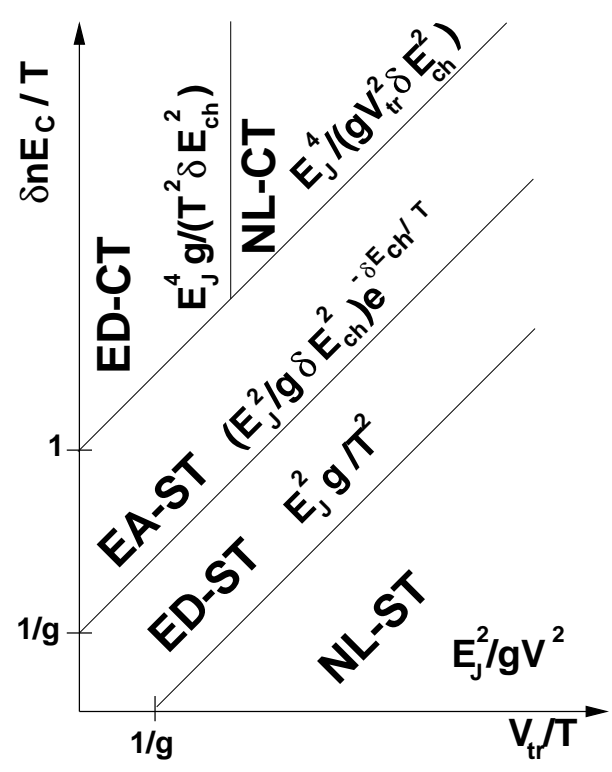

FIG. 3. Overview of the different transport regimes as a function of the transport voltage $V_{\mathrm{tr}}$ and the gate voltage (entering in the combination $\left.\delta E_{\mathrm{Ch}, 0}=-\left(n-n_{\mathrm{G}}-1 / 2\right) 4 e^{2} / C\right)$. ED-CT: Environment-dominated cotunneling; NL-CT: Nonlinear cotunneling; EA-ST: Environment-assisted sequential tunneling; ED-ST: Environment-dominated sequential tunneling; NL-ST: Nonlinear sequential tunneling

we concentrate on the high temperature limit. At the peak and for $T \gtrsim E$ we expand in powers of $\omega / 2 T$ to obtain

$$
K(t)=-2 \sqrt{\pi|t| / g C_{\mathrm{sq}}}(4|t| T-i \operatorname{sign}(t))
$$

which is dominated by the $|t|^{3 / 2}$ term. Then

$$
P(0)=\Gamma(2 / 3) / 3 \cdot\left(C_{\mathrm{sq}} g / 2 \pi T^{2} e^{2}\right)^{1 / 3} .
$$

The response of the system is again linear, but the condutance scales with new exponents: $G \propto T^{-5 / 3}$ and $G \propto g^{1 / 3}$.

If the SSCT is driven into the normal state, its own dynamics becomes dissipative, but it is still strongly influenced by the environment. In contrats to the resonant tunneling situation of the superconducting state we have to sum now over the normal final states. In this case the tunneling rate is found from $P(E)$, eq. 5 along the lines of 12.

$$
\begin{aligned}
\Gamma\left(\delta E_{\mathrm{ch}}\right) & =-\frac{2 T}{R}\left(e^{-\gamma} 2 \pi T R C_{\mathrm{j}}\right)^{2 / g} \times \\
& \times \operatorname{Re}\left[e^{-i \pi / g} B\left(1+\frac{1}{g}-i \frac{\delta E_{\mathrm{ch}}}{2 \pi T},-1-\frac{2}{g}\right)\right] .
\end{aligned}
$$

It depends on the energy again through $\epsilon$. Since the charge carriers are now normal electrons, $g$ is redefined accordingly as $g=h / e^{2} R$ and the junction strength is 

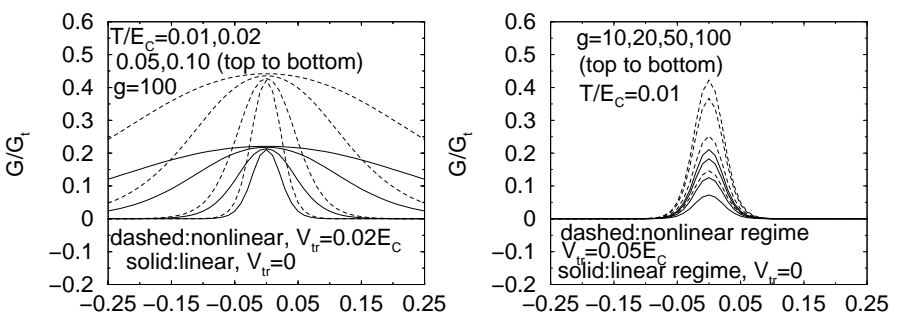

FIG. 4. Coifdifetance peaks in lowest ordef ${ }^{\delta}$ of $E_{\text {Ferturbation }}$ theory for the normal state, scaled by the tunneling conductance $G_{\mathrm{t}}$ of the junctions.

characterized by the tunneling resistance $R_{\mathrm{t}}$. For $\epsilon \ll 1$ we find $\Gamma_{\mathrm{L}}=\left(T / R_{\mathrm{t}}\right)\left(1+\delta E_{\mathrm{ch}} / T\right)$ for $g \rightarrow \infty$. Thus at the peak the response is linear again, but this time with a temperature independent value as $T \rightarrow 0$. For $\epsilon \gg 1$, the rate displays the usual Coulomb blockade

$$
\Gamma\left(\delta E_{\mathrm{ch}}\right)=\theta\left(\delta E_{\mathrm{ch}}\right) \frac{\delta E_{\mathrm{ch}} g}{\pi R_{\mathrm{t}} \Gamma(2+2 / g)}\left(e^{-\gamma} \delta E_{\mathrm{ch}} R C_{\mathrm{t}}\right)^{2 / g} .
$$

The change of the power-laws can be understood by the additional phase space factor $\propto E^{2} / T^{2}$ in the normal state. The resulting independence of the environment is caused by the fact that the system is dissipative by itself and the external dissipation is weak at large $g$. Our numerical results in Fig. 4 show the (weak) dependence on the environment conductance in the $T=0$-limit.

We are now ready to review the experimental results of the Berkeley group [7]. They constructed the SSCT just like that of Fig. 1. The parameters were: $E_{J}=1$ $\mathrm{K}, C_{t}=0.3 \mathrm{fF}, C_{0}=1.5 \mathrm{fF}$. The backplane conductance was swept in the range $g=1 \ldots 100$ and the temperature in the range $T=20 \ldots 1000 \mathrm{mK}$. They found in the Coulomb oscillations of the conductance a linear response regime at the peaks, confirming a major prediction of the present theory. The peak conductance was found to scale as $G \propto g^{0.3}$ and $G \propto T^{-0.9}$. Their lead arrangement is modeled more adequately with a transmission line. Our relevant results for this case are: $G \propto g^{1 / 3}$ and $G \propto T^{-5 / 3}$. When the SSCT was driven normal by a large magnetic field, they found that the peak conductance exhibits very weak dependence on $g$ and $T$. Our theory predicted that $G$ goes to a constant for either large $g$ or small $T$. So several of the measured $g$ and $T$ dependences are in agreement with the present theory. Finally, between the peaks they do not find an exponential suppression of $G$, making it plausible that indeed the cotunneling process is the dominant transport channel, as described above.

A similar system, a superconducting electron box mounted on a metallic ground plane [16], has been used to demonstrate the coherent oscillations of charge states [3], which makes this setup a promising candidate for a qubit in quantum computers. To this end, it has to be operated at the peak. The quality factor $Q$ of the oscillations can be determined by standard methods [1]. At the low temperatures of $T \ll E_{\mathrm{J}}$ we obtain

$$
Q=\cot (\pi /(g-2)) \approx g / \pi
$$

as for the metallic case, $g \gg 2$. For the parameters of [3] we find $Q$ in the range of several hundreds. Thus the dissipation allows of the order of a thousand cycles of the quantum oscillations. While this is encouraging, it still shows that the metal planes, which were introduced to screen the random charges, induce a potentially serious limitation on the maximum number of coherent operations.

Numerous stimulating discussions with J. Clarke, T. Giamarchi, L. Glazman, C. Kurdak, R. Therrien, J.B. Kycia, J. Chen, J. Siewert, G. Falci, J. König, and A.D. Zaikin are acknowledged. The work at UC Davis was was supported by NSF-9720440, NSF-DMR 9985978 and by NATO 971615. FKW and GS are supported by the DFG through SFB 195 and GK 284 and by the EU through TMR Superconducting Nano-circuits. GTZ's work at the UCSB ITP has been supported by NSF-PHY-94-07194.

[1] A.J. Leggett et al, Rev. Mod. Phys. 59, 1 (1987).

[2] Yu. Makhlin, G. Schön, and A. Shnirman, Nature 398, 6725 (1999).

[3] Y. Nakamura, Yu.A. Pashkin, and J.S. Tsai, Nature 398, 786 (1999).

[4] A.J. Rimberg et al., Phys. Rev. Lett. 78, 2632 (1997).

[5] L.S. Kuzmin, Yu.V. Nazarov, D.B. Haviland, P. Delsing, and T. Claeson, Phys. Rev. Lett. 67, 1161 (1991).

[6] K.H. Wagenblast, A. van Otterlo, G. Schön and G.T. Zimányi, Phys. Rev. Lett. 79, 2730 (1997).

[7] C. Kurdak et al., Physica E 6, 825 (2000). J.B. Kycia et al., submitted to Phys. Rev. Lett.

[8] K.A. Matveev, M. Gisselfält, L.I. Glazman, M. Jonson, and R.I. Shekter, Phys. Rev. Lett. 70, 2940 (1993).

[9] P. Joyez, P. Lafarge, A. Filipe, D. Esteve, and M.H. Devoret, Phys. Rev. Lett. 72, 2458 (1994).

[10] H. Grabert, G-L. Ingold, and B. Paul, Europhys. Lett. 44, 360 (1999).

[11] M.H. Devoret, D. Esteve, H. Grabert, G.L. Ingold and H. Pothier, Phys. Rev. Lett. 64, 1824 (1990).

[12] G. Ingold and Yu. V. Nazarov in Single Charge Tunneling, NATO ASI Series, Vol. B 294, edited by H. Grabert and M. H. Devoret (Plenum Press, New York, 1992).

[13] D.V. Averin, Yu.V. Nazarov, and A.A. Odintsov, Physica B 165\&166, 945 (1990).

[14] Quantum Transport and Dissipation, T. Dittrich et al., Wiley-VCH Publ. (1998); Chapter 3, p. 149-212 and references therein.

[15] A.A. Odintsov, V. Bubanja, and G. Schön, Phys. Rev. B 46, 6875 (1992).

[16] Y. Nakamura and J. Tsai, J. Low Temp. Phys. 118, 765. 